\title{
A NEW INDOOR LiDAR-BASED MMS CHALLENGING COMPLEX ARCHITECTURAL ENVIRONMENTS
}

\author{
C. Bonfanti ${ }^{1}$, G. Patrucco ${ }^{2}$, S. Perri ${ }^{2}$, G. Sammartano ${ }^{2,3}$, A. Spanò $\grave{2,3}^{2,3}$ \\ ${ }^{1}$ CAM2 (FARO Technologies, Inc.) AEC Team Italy \\ ${ }^{2}$ DAD - Department of Architecture and Design / Politecnico di Torino, Viale Mattioli 39, 10125, Torino (Italy). \\ giacomo.patrucco@polito.it; stefano.perri@polito.it; giulia.sammartano@polito.it; antonia.spano@polito.it \\ ${ }^{3}$ FULL - Future Urban Legacy Lab, Politecnico di Torino. Via Agostino da Montefeltro 2, 10125 Torino (Italy).
}

KEY WORDS: 3D models, rapid mapping, LiDAR, MMS, SLAM, accuracy evaluation, SWIFT system by Faro Technologies

\begin{abstract}
:
The use of moving devices equipped with range- and image-based sensor, generically defined Mobile Mapping systems (MMS), have been quite a disruptive innovation in the development of Geomatics techniques for 3D surveying large indoor-outdoor spaces and offer multiple solutions. The recent expansion of portable devices in the form of trolleys, backpacks, handheld tools largely implements SLAM (Simultaneous Localization and Mapping) algorithms and technology based on both Lidar and/or visual solutions for answering to the positioning and the 3D reconstruction problems. The research on MMS is directed to improve both multi-sensor integration implementation and usability of systems in diversified use contexts and application fields. The aim of the presented research is the evaluation of the potential of the Swift system recently developed by FARO Technologies, that has been fine-tuned for regular and large extent interiors mapping (such as factories, hospitals, airports, offices). The work tries to preliminary investigate the data delivery and usability of the integrated system. This is based on three elements mounted on a sliding trolley moved by the operator walking: the ScanPlan profilometer working for the 2D SLAM mapping, the static TLS Focus S-series, and the smartphone managing the sensors operation and the acquisition progress. The evaluation strategy undertaken will be based on the global and local performance analysis related to the trajectory, the data accuracy, the metric content and consistency. Two test studies belonging to the $20^{\text {th }}$ century. architecture are presented in a preliminary framework of evaluation and validation: a Liberty-style cinema and the Torino Esposizioni Hall B designed in ferrocement by pier Luigi Nervi.
\end{abstract}

\section{INTRODUCTION}

The use of 3D models and geospatial data derived from dense clouds by applying geomatic techniques has certainly undergone rapid development and increasingly widespread use in many fields of application. Many scientific communities of the most varied fields of study are increasingly estimating the relatively easy and fast availability of 3D models on which to base - or to be able to support in different ways - investigations and researches of their interest (Betti et al., 2021).

It is in fact quite recurrent that the research for efficient solutions has considerably shifted to the so-called Digital Twins. Such models are enriched by the possibility of interacting with heterogeneous information or external databases, allowing the exchange of information and analyses also in the form of provisional ones with benefits among specialists of different training and operating on objects of interest with different purposes. Therefore, rapid acquisition and accuracies, level of details, reliabilities adequate to the degrees of analysis and representation are increasingly required, to lighten the preliminary phases of data collection and model generation to shift attention to the subsequent phases of analysis.

As generally happens in the field of documentation of architectural heritage, even of large complexes (Barba et al., 2021; di Filippo et al., 2018; Remondino et al., 2009), the demand and the interest in the use of multi-scale and multi-content models - and therefore of course multi-sensor - is current even when there is a structural interest in these assets.

Here, in fact, the ability to obtain 3D models is crucial. This can provide a general vision of the structure and the possibility of investigations at different levels of detail, especially where the structural degradation is investigated. In this regard, it is very interesting and actual the possibility to derive lightweight models for subsequent processing or predictions of the structural behaviour.

After a brief overview of the latest innovations on systems capable of conducting rapid data acquisitions, especially in mobile configuration, the study will introduce the use of a new device (the Swift system by FARO Technologies), which has been tested in two selected cases in order to highlight the advantages and any critical issues of use. The two case studies belong to the twentieth century Piedmont architecture, but they are very different in the challenges they pose to the new data acquisition system. The first one is the foyer of the Politeama cinema in Ivrea, characterised by typical architectural liberty style details. The second one is the large Torino Esposizioni complex (Hall B) in Turin (Italy) by Pier Luigi Nervi. It presents eminent spaces enclosed by vaulted solutions typical of the pioneering development of the use of ferrocement in the " 50 s of the twentieth century. Both case studies offer the possibility of evaluating the data quality and a truly wide range of modelling skills, assessing the degree of accuracy response to the specific needs and an overall performance assessment of the new mobile mapping system proposed by FARO Technologies.

\subsection{D (mobile) mapping technologies framework}

In the framework of imaging and ranging measurement systems for 3D data acquisition, a previous season has certainly demonstrated the effectiveness of integrating techniques.

More recently, a dizzying development of fusion-based sensors solutions raised, originating a different series of systems born in the field of rapid mapping technologies based on mobile strategy. A first development of MMS (Mobile Mapping System) is based on 
sophisticated and pricey systems that integrated a LiDAR sensor or active 3D imaging devices as mapping unit, an inertial measurement unit (IMU), a GNSS (Global Navigation Satellite System) receiver and a time referencing unit (Puente et al., 2013). They are often equipping moving vehicles but in recent times many portable systems were developed also in the form of trolleys, backpacks and scanner cart (TIMMS system by Trimble - https://www.trimbleitalia.com/prodotti/mobile-mapping/trimble-timms and ProScan platform by Leica - https://leica-geosystems.com/itit/products/mobile-sensor-platforms/capture-platforms/leicaproscan, for example). More recently, the seamless positioning problem has been pursued using new strategies. An emerged solution is surely the SLAM (Simultaneous Localization and Mapping) technology that has been integrated in many portable solutions: handheld systems (Zeb Revo by GeoSLAM and BLK2go by Leica geosystems are two examples). The techniques of mobile mapping based on various implementations of the SLAM methods (Søren Riisgaard \& Morten Rufus Blas, 2005) exploit data acquired by a LiDAR sensor and are capable to determine the 3D position of the device during the connected trajectory. Thanks to the geometric characterization of the setting, the system is able both to estimate the movement of the sensor and the progressive alignment of 3D clouds data of the mapped space according to an Iterative Closest Point (ICP) algorithm. These systems have been studied and tested in many different fields of applications, in indoor and outdoor environments in comparison with more consolidated methods (Masiero et al., 2017; Nocerino et al., 2017; Sammartano \& Spanò, 2018; Tucci et al., 2018).

Solutions that pursue similar objectives have been studied using the so-called laser-visual-inertial odometry (Zhang \& Singh, 2018). It appeared on the market through the development of the Kaarta STENCIL sensor which combines a 3D LiDAR sensor with a camera for features correlation and an inertial platform to map in real time and without the use of GNSS receivers outdoor and indoor environments even of considerable size (Calantropio et al., 2019; Di Pietra et al., 2020). Manageability and rapidity in acquisition for 3D reconstruction of complex scenes are also pursued by traditional TLS (Terrestrial laser scanning) solutions that are now able to speed up the cloud registration using an automated targetless field registration based on VIS (Visual Inertial System) technology (Fuentes-Pacheco et al., 2012). In this rich and complex scenario with very many different and end-user-oriented solutions, the new Swift scanner cart integrated system by FARO Technologies, is fine-tuned for repetitive vast indoor environments. The paper focuses on some tests in complex and challenging environments with the purpose to evaluate the effectiveness of the new system based on SLAM technology too.

\section{THE SWIFT SYSTEM BY FARO}

\subsection{Swift: an integrated technology}

The potential of the Swift system by FARO Technologies is based primarily on its integrated nature. In fact it is built up of three different connected components (Figure 1a):

a 3D static laser scanner of the $\mathrm{S}$ series (also working independently);

the so-called ScanPlan equipped with a laser profiler through which the SLAM function works (also working independently as profilometer and 2D floor fast plan generator);

the smartphone connecting in a Wi-Fi mode the two sensors. The system operation is controlled via GUI from smartphone browser interface.

All the components are placed in a light and stable trolley pushed by the operator walking during the survey (Figure 1b). The profile sensor, characterised by an operative range of up to $20 \mathrm{~m}$ (but an updating working range has been recently foreseen to $60 \mathrm{~m}$ ), detects features in a plane that are used by SLAM function in order to estimate the trajectory during the mapping phase. The LiDAR range performance depends on the static $\mathrm{S}$ series capabilities (the longrange S350 is the one tested here). The amount of data collection is thus correlated to the scanner performance and to the acquisition speed. The system has been fine-tuned for large extent interiors mapping by scanning (such as factories, hospitals, airports, offices). Concerning the costs, it is necessary to notice that the swift system is an extension of static scanners, with a discrete advantage obviously for users who already have an S series FARO scanner (the total amount is about some tens of thousands euros depending on national market).
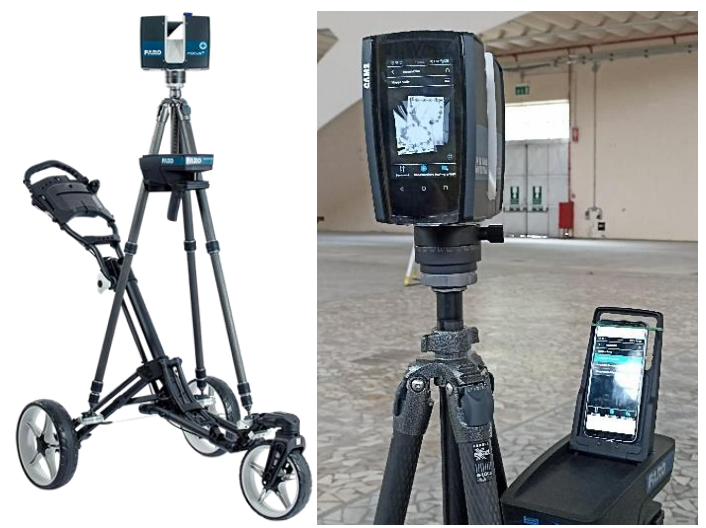

(a)

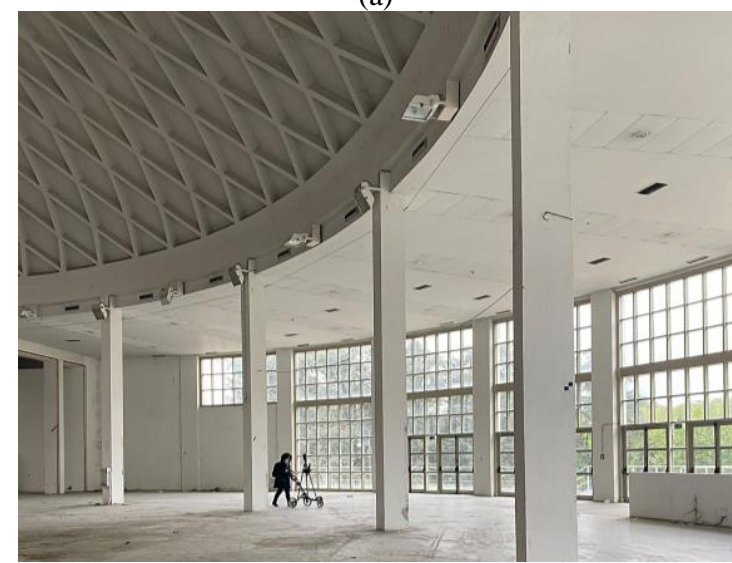

(b)

Figure 1. The components of the Swift System by FARO Technologies (a). A view of the acquisition phase in the Torino Esposizioni Hall B.

\subsection{Principles of operation}

The system operates in three scanning modes: the mobile continuous scanning; the short-static anchor scans, and finally ordinary static scans. The acquisition speed of the mobile scanning is linked to the slow pace of the operator pushing the trolley; it is difficult to indicate in absolute terms the time savings compared to the use of the static technique, as it largely depends on the configuration of the environment to be mapped. Surely, it is possible to acquire scanning sessions lasting about half an hour. If anything, it is necessary to carefully evaluate the balance between scan duration and consequent file size, the heaviness of which affects the subsequent processing times.

The anchor scans, that are carried out along the trajectory keeping slowing and performing a short static-mode scan, are supposed to be acquired in critical steps of the scanning session, when there are 
problematic places in the scanning environment such as long corridors, crossing door among different rooms, curved lines etc. The usual static scans are the only ones that can be activated in order to start the acquisition of the images for radiometric data; their role is also aimed at post-processing better evaluation of positioning issues and levelling control. The tests performed in this research underline the necessity of avoiding stairs and ramps although with an insignificant slope, and this confirms the best practice advices from the FARO Technologies distributing the system. Also reversing the trajectory should be avoided. At the moment the use is recommended for indoors environments, since the scan can be affected by serious problems if the floor is not smooth and there is an even slight disturbance of uncovered floor, as we have been able to ascertain in an underground environment not reported in this paper. The system is evolving rapidly and very recently further option have been offered to the user to manage the different types of scans separately, i.e. mobile scans and anchor scans as independent objects, and also in relation to the time relating to the different acquisition phases. Furthermore, recent updating implemented in latest releases will make available the possibility of managing the information relating to the time for the trajectory and consequently for the point clouds (a new test has been done recently).

\subsection{Processing and registration strategies}

The registration process is currently semi-automatic and it takes place in two time-steps: first, the SLAM algorithm operates simultaneously with the processing of the mobile scans that are joined together on the basis of the position of the subsequent projective centres and collected in the trajectory file, and second, into the SCENE software interface, with procedures of co-registration and accuracy check, as deepened in following paragraph. The anchor scans and the static scans with radiometric data complete the procedural acquisition mode helping the registration phase. Spherical targets can be also used during the scanning acquisition and can be exploited in the SLAM processing to enhance the scans alignment. Some recommendations for scanning performances are compliant with SLAM mapping requirements in the data capturing phase: finalising the mobile scans at the original position in order to increase geometrical constrains to help the processing; stopping briefly the scans when the scene changes suddenly, for example when passing a door, to collect overlapping areas in some parts of the trajectories to increase again $3 \mathrm{D}$ feature matching and geometric constrains for the positioning problem solution, etc. Other requirements are linked to the operative functioning of the profile scanner intended to keep the trajectory under control: flat floor and ceiling, presence of enough features in the field-of-view of the profiler scanner, at proper distance from the profilometer; slope and stairs cannot be scanned with the trolley during a scan trip but may be integrated by the static method.

\section{CASE STUDIES TESTS}

The two experimental tests focused on different purposes, which can be summarised as follows in par. 3.1.1 and 3.1.2. The early 20th century cinema in Ivrea and the Turin Exhibition Centre halls by Pier Luigi Nervi are surely two distinct examples of spaces with relevant features and challenging variables to make effective and consistent the experimentation of the mobile mapping system and the trajectory performances. Not only the variables related to the use of the system technology itself but also the metric characterization of the point clouds results will be presented and discussed in the following paragraphs according to the peculiar environmental conditions. A specific attention will be dedicated to Torino Esposizioni Halls.

\subsubsection{The 3D mapping of the $\mathrm{XX}$ century cinema}

The Liberty style of the cinema (Figure 2), the staircase leading to the gallery, the specificities of the furnishings pertaining the beginning of the past century, make the site an example of interesting interior architecture requiring high details to describe the morphological and decorative thin details and strong radiometric characterization, together with high accuracy and cloud density. The slightly inclined parterre hall of the cinema represents a crucial issue in the results of the scan processing and SLAM performance. The cinema with the foyer visible in Figure 2a, the parterre and the gallery are, as introduced, a specific test site in a particular enclosed environment, with the dimensions of almost $30 \mathrm{~m}$ long, $12 \mathrm{~m}$ wide and $9 \mathrm{~m}$ high (Figure 2b). Here the Swift system needed separated trajectories in the different spaces.

The Swift continuatively acquired inside the 3 spaces but it was not able to collect along stairs elements, due to its motion system, of course. Also in the parterre room the configuration of pavement introduced a variable element challenging the SLAM working with the Scan Plan. For this reason, a set of static scans have been acquired for the rigidity of the geometric configuration and for the radiometric data acquisition too (see par. 4.2.1)

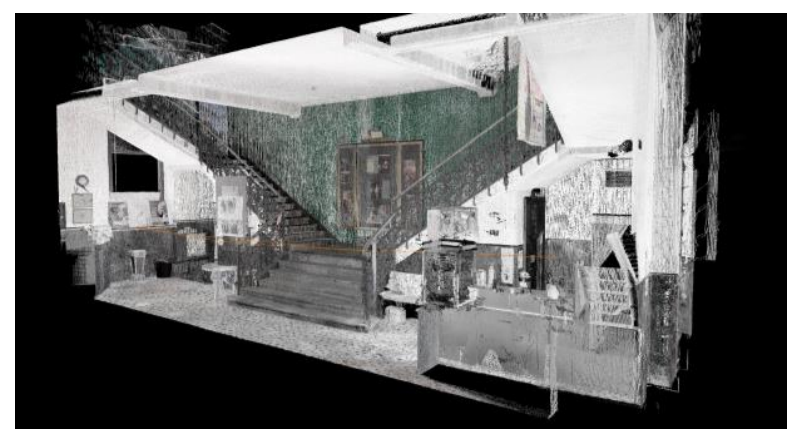

(a)

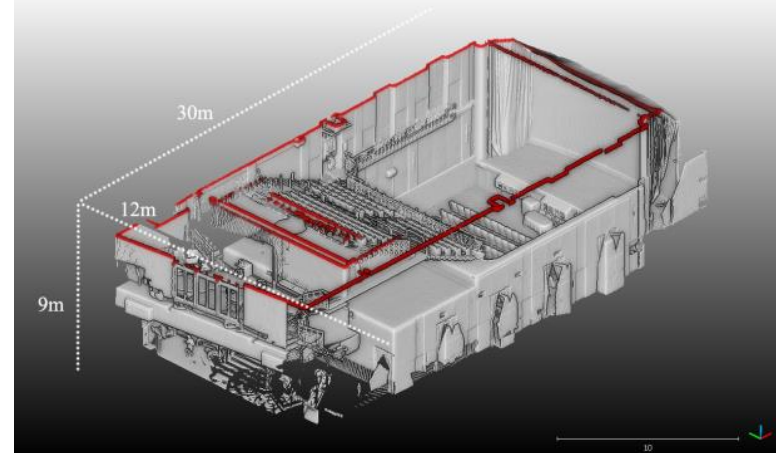

(b)

Figure 2. The preview of the Swift data on the XX century cinema, with indication of the main dimensions.

\subsubsection{The Nervi's Torino Esposizioni Halls}

The Turin Exhibition Centre halls by Pier Luigi Nervi, recognised as one of the most relevant $20^{\text {th }}$ century architectural concrete asset in the world, is featured by unusual dimensions and extraordinary complexity of ferrocement geometries. Mostly, the case study of Hall $\mathrm{B}$ in Torino Esposizioni (Figure 3) and its huge extension, is a very challenging context, with the dimensions of almost $150 \mathrm{~m}$ long, $70 \mathrm{~m}$ wide and $20 \mathrm{~m}$ high, and the internal layout with high characterising features, with the corridors and the two levels structures with cantilevers and slanted pillars.

Furthermore, at the moment of the survey the huge hall was occupied by temporary equipment used for the exhibition purposes of the site, distributed in a rather labyrinthine way, this requiring an adaptation of the planning of the acquisition strategies, according to the accessibility of the spaces. In fact, such bulks, in the case of the adoption of the traditional laser scanning technique would cause the need to deploy a lot of scanning positions. 
The Swift system proved the capability to be a challenging solution for that, not only for saving time, taking advantage of traveling the wide and high hall spaces. In addition to the ability of returning the architectural ensemble of the vast room, the Swift system is also efficient in digitising the details of the structural elements (slanted pillars, cantilevers elements, undulated thin shell vault etc.) in the consequent 3D model.

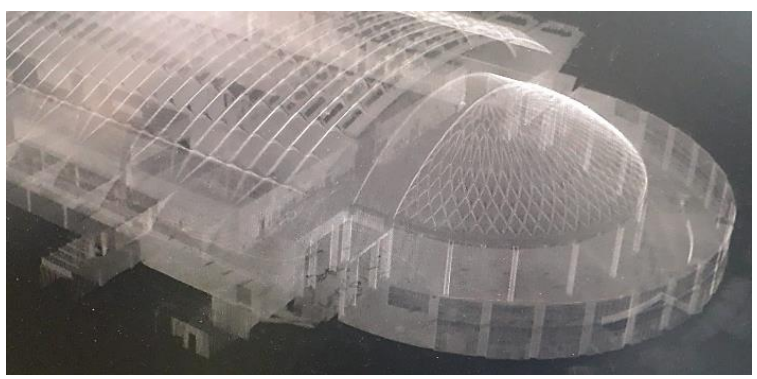

(a)

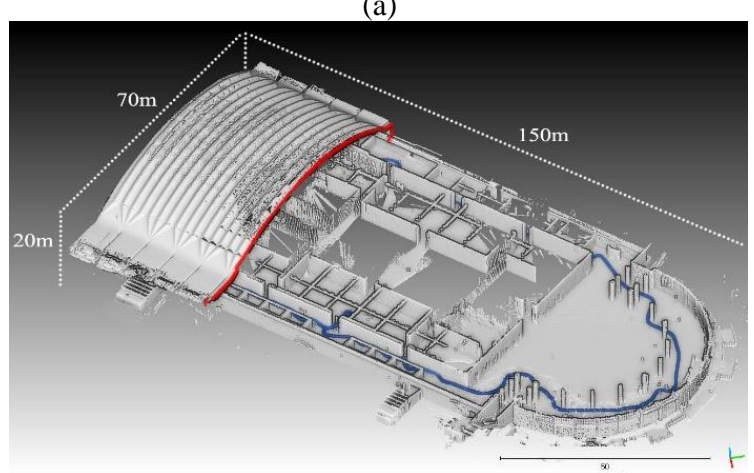

(a)

Figure 3. The preview of the Swift data on the Torino Esposizioni, hall B, with indication of the main dimensions.

Table 1. Synthetic description of the three trajectories

\begin{tabular}{|c|c|c|c|}
\hline & A & B & C \\
\hline Time & $12 \mathrm{~min}$ & $10 \mathrm{~min}$ & $10 \mathrm{~min}$ \\
Length & $411.36 \mathrm{~m}$ & $389.673 \mathrm{~m}$ & $181.435 \mathrm{~m}$ \\
$\mathrm{~N}^{\circ}$ points & ca. $705 \mathrm{mln}$ & ca. $566 \mathrm{mln}$ & ca. $304 \mathrm{mln}$ \\
Loop closure & Yes & Yes & Yes \\
Overlapping & No & Yes & Yes \\
Static scans & No & No & No \\
Anchor scans & No & No & Yes \\
\hline
\end{tabular}

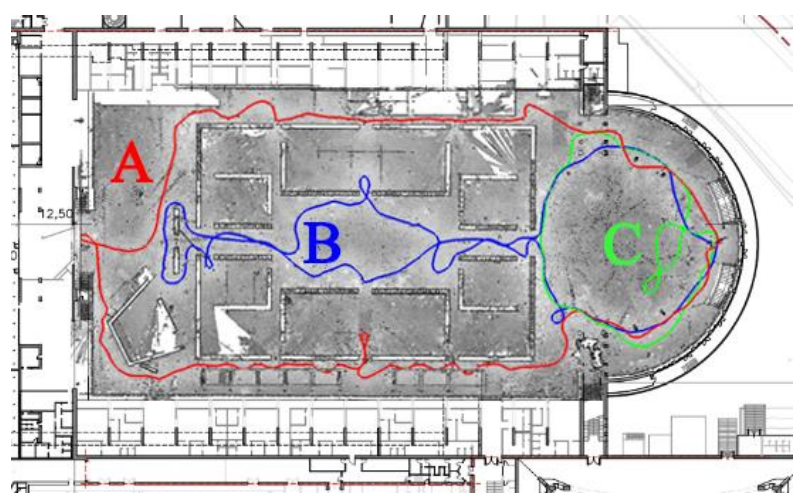

Figure 4. The three trajectories performed in the B Hall

Here the reference dataset as ground-truth is a static Lidar acquisition. $\mathrm{N}^{\circ} 17$ scans have been collected using a Faro Focus ${ }^{3 \mathrm{D}}$ X330 phase shift laser scanner: $\mathrm{n}^{\circ} 7$ scans have been acquired in the first sector of the pavilion hallway, while $n^{\circ} 10$ scans have been acquired in the exedra apse. The point clouds have been registered by blocks using the Faro Scene software interface. Firstly, a ICP (Iterative Closest Point) registration has been conducted exploiting the rich geometric features of the overlapping scans: here the mean error after this preliminary alignment is $\approx 2 \mathrm{~mm}$. Then, the two blocks have been rigidly co-registered with an accuracy check on control points, using a set of $n^{\circ} 30$ contrast markers measured using a total station. In this second step the verified accuracy on markers is $\approx 4.5$ $\mathrm{mm}$ (mean deviation between the markers in the point cloud and the measured points). Three different trajectories have been performed as a strategy to preliminary evaluate three different point clouds results. They are indicated in the Figure 4 plan and the main features are listed in Table 1 . The operator completed a first path (A) in the central hall within the corridor external to the movable installation panels, the longer with $\mathrm{XX}$ points, closing the loop and without retracing roundtrip. The (B) path passes in the internal corridor of the movable partitions, making a roundtrip and closing in the starting point. The (C) acquisition was focused on the vaulted and pillar space of the exedra. A set of spherical 3D markers, as introduced, were distributed and the trajectories always tries to follow this objects distribution. The trajectories delivered several hundreds of million points per scans within a scans time of 10-12 minutes walking. The three scans have been registered using spheres.

\section{RESULTS DISCUSSION ON METRIC EVALUATION}

The evaluation strategy undertaken is based on a double analysis, from a global- and a local-scale metric assessment, as frequently conducted by research works in case of SLAM mobile mapping acquisitions typically affected by a double trend behaviour (Nocerino et al., 2017; Sammartano \& Spanò, 2018; Toschi et al., 2015; Tucci et al., 2018). On one hand, the evaluation of the SLAM datasets and the associated trajectories are based on the accuracy performance using as ground-truth the 3D scans planning acquired by static TLS. In this case a particular attention should be paid to the point cloud co-registration and comparison since the two datasets presents specific configurations and different coverage homogeneity and mostly since the two sensor have its own precision. Furthermore, the Swift scan dataset was very huge and challenging to be managed together with the static scans project. The essential goal is to estimate the uncertainty of the resulting 3D data considering the evaluation of the overall accuracy, especially in terms of 3D space reconstruction and drift errors affecting the trajectory according to the diverse environment conditions. This is implemented in these preliminary results using statistical approach by evaluation of RMSE based on deviation distances and residuals errors check using control points, distance measurements and ICP based cloud-to-cloud alignment (Par. 4.1). On the other hand, the preliminary evaluation of the point cloud is conducted also in a local-scale perspective, analysing the points surface characterization based on the geometric and radiometric content discussion. The results validation has been organised deepening the geometrical accuracy of surface reconstruction, that will be presented, according to points clouds 3D features (es: neighbour density, noise by roughness index).

\subsection{Global performance and accuracy validation in Hal B}

Here the global performance of the Swift system is presented using the Torino Esposizioni dataset. The consistent evaluation of the global accuracy on the point cloud geometry derived from the Swift scanner mapping requires some reflection on the different approaches to follow considering many aspects of the assessment occurring in the concept of global accuracy. In the case of the global assessment, it means the quality of the measurements of the $3 \mathrm{D}$ reconstruction of the entire space, considering a double combined behaviour, the metric one related to the range sensor 
with the mobile solution, and the SLAM functioning progressively co-registering the seamless scans acquisition.

A first usual accuracy evaluation consists in semi-automatic check with markers and ICP, using Scene SW. This procedure followed multiple steps, involving both the mobile and the static datasets: the scans registration benefits from both the 3D spheres and the topographic measured markers.

In the first step the (A), (B) and (C) mobile scans have been coregistered based on the spherical targets with a mean error value of $0.004 \mathrm{~m}$ on spheres located in the abse area and of $0.02 \mathrm{~m}$ in local controls at the opposite side of the hall to verify deviation due to different trajectories A and B ([1 $\left.1^{\text {st }}\right]$ block). The static ones have been registered and checked with topographic markers, with a mean error value on the points set of $0.045 \mathrm{~m}$ ([2 $\left.2^{\text {nd }}\right]$ block). After the coregistration of $\left[1^{\mathrm{st}}\right]$ and $\left[2^{\text {nd }}\right]$ blocks, the final overall accuracy of the mobile dataset results measured on markers used as reference points is a residual deviation error of $0,037 \mathrm{~m}$.

Moreover, a comparison based on cloud-to cloud co-registration and deviation analysis of the final MMS clouds with the ground truth has been conducted and delivered a result of ICP $98 \%$ points $< \pm 2 \mathrm{~cm}$. The substantial difference between the two clouds overlap does not allow to fully appreciate this result, which does not consider metrically all the error distribution. In addition to the ICP method, a point-based co-registration has been performed and an accuracy control has been proposed using $\mathrm{n}^{\circ} 20$ homologous points well distributed on the whole building and recognised on both the datasets (TLS and MMS), with a mean of deviation error $=0.101 \mathrm{~m}$, st.dev.$=0.038 \mathrm{~m}$.

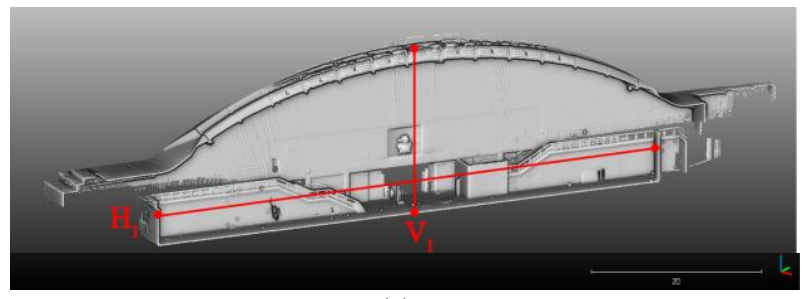

(a)

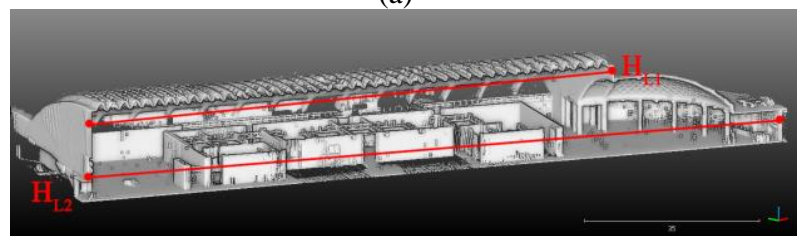

(b)

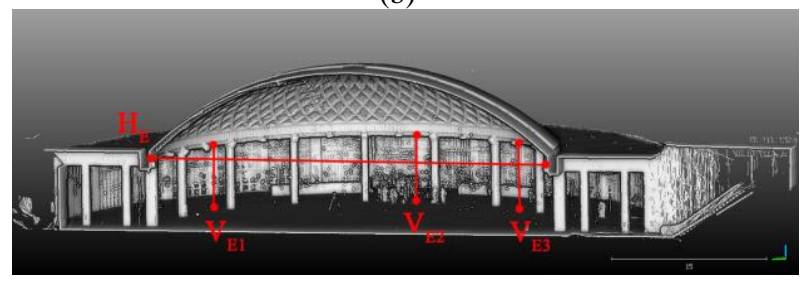

(c)

Figure 5. The indication of the distance measurements

Finally, a performance evaluation on the global 3D reconstruction has been based on a sample of recurring measured distances.

This set of distance measures have been selected, as indicated in Figure $5 \mathrm{a}, \mathrm{b}, \mathrm{c}: \mathrm{n}^{\circ} 4$ vertical and $\mathrm{n}^{\circ} 4$ horizonal absolute distances, and each measure has been repeated for 10 times in order to collect redundant measures and to extract a mean value of the distances and an absolute residual error between the MMS data and the ground-truth TLS dataset. As visible in Table 2, the analysis of punctual distances in vertical direction stands on mean values of residuals between 3 and $5 \mathrm{~mm}$, with $\sigma=2-3 \mathrm{~mm}$.
The distances most affected by drift error are as expected those longitudinal, $\mathrm{H}_{\mathrm{L} 1}$ and $\mathrm{H}_{\mathrm{L} 2}$, i.e. in the direction of the length of the trajectory, where the error achieves the values of $46 \mathrm{~mm}$.

Table 2. Discrepancies between TLS- and Swift-derived measured distances, performed on the point clouds using both horizonal and vertical directions, on a statistic sample of 10 repetitions

\begin{tabular}{|c|c|c|c|c|c|}
\hline & & $\begin{array}{c}\text { Mean } \\
d_{T L S}(m)\end{array}$ & $\begin{array}{c}\text { Mean } \\
d_{M M S}(m)\end{array}$ & $\begin{array}{c}\text { Res. } \\
\text { mean }(m)\end{array}$ & $\underset{(m)}{\sigma}$ \\
\hline \multirow{2}{*}{ (a) } & $\mathrm{H}_{\mathrm{I}}$ & 64,947 & 64,948 & 0,003 & 0,002 \\
\hline & $\mathrm{V}_{\mathrm{I}}$ & 18,373 & 18,375 & 0,003 & 0,003 \\
\hline & $\mathrm{H}_{\mathrm{L} 1}$ & 137,490 & 137,536 & $0,046^{*}$ & 0,013 \\
\hline & $\mathrm{H}_{\mathrm{L} 2}$ & 112,404 & 112,443 & $0,040^{*}$ & 0,009 \\
\hline \multirow{4}{*}{ (c) } & $\mathrm{H}_{\mathrm{E}}$ & 39,516 & 39,512 & $0,011^{*}$ & 0,005 \\
\hline & $\mathrm{V}_{\mathrm{E} 1}$ & 15,636 & 15,639 & 0,003 & 0,001 \\
\hline & $V_{E 1}$ & 6,549 & 6,548 & 0,004 & 0,003 \\
\hline & $\mathrm{V}_{\mathrm{E} 1}$ & 6,545 & 6,544 & 0,005 & 0,002 \\
\hline
\end{tabular}

\subsection{Local evaluation and geometric characterization}

After the considerations of the previous sections, a set of analyses has been carried out to locally evaluate the features and the principal characteristics of the Swift point clouds, starting from the radiometry and the geometry of a single scan: for this preliminary evaluation, the point cloud acquired in the foyer, for the cinema and the scan following the trajectory $\mathrm{C}$ for Torino Halls (Figure 4) has been considered.

\subsubsection{The radiometric data for the cinema foyer}

Moreover, it is possible to evaluate the radiometric content of the global acquisition. Here we reported the $1^{\text {st }}$ case study since it is more effective from the decorative point of view. The main difference for the colour rendering of the radiometric data is surely the quality of the camera, between the Faro Focus ${ }^{3 \mathrm{D}}$ X330 (70 MPX HDR camera) and Faro Focus ${ }^{3 \mathrm{D}}$ S350 equipping the Swift MMS (colouring with 165 MPX HDR) (Figure 6). As a consequence, the homogeneity of the radiometric information is function not only of the density but also of the continuity of data, as for the Swift dataset in Figure $6 b$.

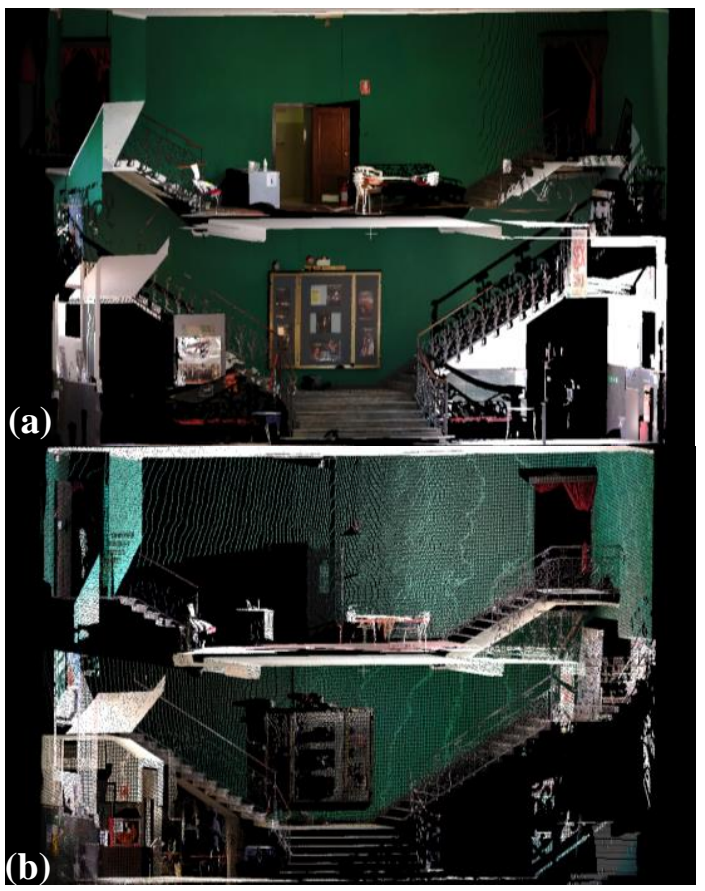

Figure 6. The colour appearance comparison between static LiDAR (a) and MMS (b). 


\subsubsection{Density analyses along the trajectory}

A density analysis has been computed on the point cloud acquired in the apse of Hall B using the number of neighbours method: the density has been estimated considering - for each point - the neighbours inside a sphere with a radius of $0.02 \mathrm{~m}$ (Figure 7). The software used to achieve this analysis is the open-source platform CloudCompare (CC). As predictable, by the outcome of this analysis it is evident that the density of the point cloud is highly related to the acquisition distance between the sensor and the acquired object, and therefore to the trajectory followed by the operator and to the time spent acquiring the surveyed surfaces.

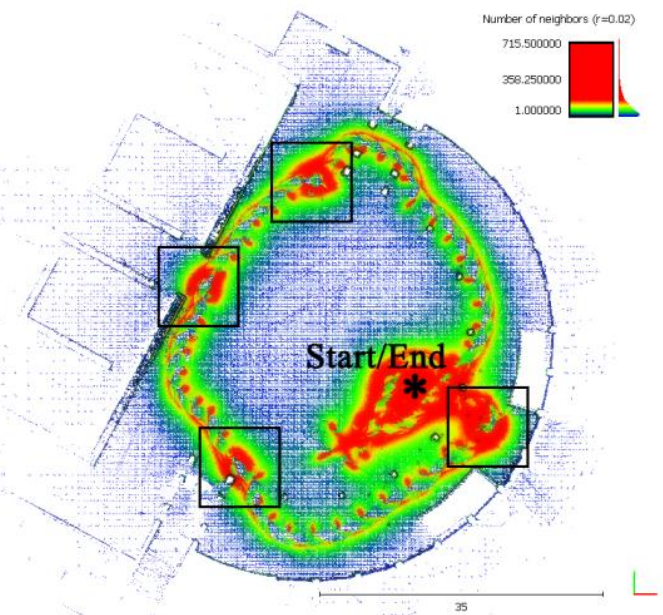

Figure 7. Density analysis of the Swift point cloud acquired in the apse. The analysis has been performed using CC (number of neighbour analysis, pts/V sphere $\mathrm{r}=0.02 \mathrm{~m}$ ). The black squares areas are the position of the spherical targets; area $(*)$ is the starting point of the acquisition.

As it is possible to observe in Figure 7, considering trajectory (C) in Figure 4, the density of the scan is significantly higher along the trajectory followed by the operator during the acquisition. In addition, a further increase of the density can be observed where the operator has stationed for a longer time (for example, as in this case, in the $(*)$ area adjacent to the starting point of the scan, or where spherical targets were positioned). The density analysis along the other trajectories also adjacent to the hall (A) and (B) in Figure 4 demonstrates a high concentration of the mobile mapping point cloud on the areas travelled by the scanner (between almost $23.000 \mathrm{pt} / \mathrm{mq}$ and $53.000 \mathrm{pt} / \mathrm{mq}$ according to the sensor distance, respectively at $20 \mathrm{~m}$ on the vault and at $1 \mathrm{~m}$ distance on the ground). Considering now the point cloud on a larger scale, three samples of the point clouds acquired in the Hall B have been selected below, to perform a comparison with the traditional static TLS scans (used as groundtruth): a portion of the higher part of the vault (the more distant areas to both the sensors used during the survey) (Figure 8 and Figure 9), a planar vertical surface (a portion of the wall in the southern apse) (Figure 10 and Figure 11) and an element of the decorative apparatus, the Giovanni Agnelli statue (Figure 12 and Figure 13), placed in the hallway of the pavilion (in this case the main goal was to evaluate the achievable level of detail on medium-small objects). The aim of analysing these particular elements was to assess the geometric features and the quality of the acquired scans from a local-scale perspective. For this reason different analyses have been carried out in this preliminary phase of the research, focusing on the evaluation of each portion. Therefore, a density analysis (number of neighbours analysis: in this case it has been considered for each point a sphere with a radius of $0.05 \mathrm{~m}$ ) and a roughness analysis (for each point a best fitting plane is computed considering the neighbours inside a sphere with a radius of $0.05 \mathrm{~m}$; the roughness value is considered as the mean deviation of the points from the estimated plane) have been performed. Also in this case the used software is CC.

\subsubsection{The apse vault's detail}

The first considered area was the upper part of the apse's vault. From Figure 8 it can be observed that the LiDAR point cloud is characterised by a higher and more homogeneous density (mean value: ca 334 points/Vsphere $\mathrm{r}=0.05 \mathrm{~m}$ ).

As regards Swift derived data (density mean value: ca 191 points/V sphere $\mathrm{r}=0.05 \mathrm{~m}$ ), the point cloud is sparser between the vault rafters, due to the longer acquisition distance and to the occlusions represented by the rafters, but thanks to the free movement of the device it can be observed a higher completeness of the final data (in comparison with the LiDAR point cloud) in the area located behind the arch.

As regards the roughness analysis which has been carried out to evaluate the level of noise in the Swift System point cloud, it is evident from Figure 9 that the level of noise is greater when compared to the traditional TLS data. In this case the mean value, as reported in the graph below, is $\approx 2 \mathrm{~mm}$ for LiDAR and $\approx 5 \mathrm{~mm}$ for the Swift system. Besides, a stripe pattern begins to be visible on the acquired surface indicating the acquisition profile of the laser beam of the employed device (the pattern becomes more evident in the subsequent examples).
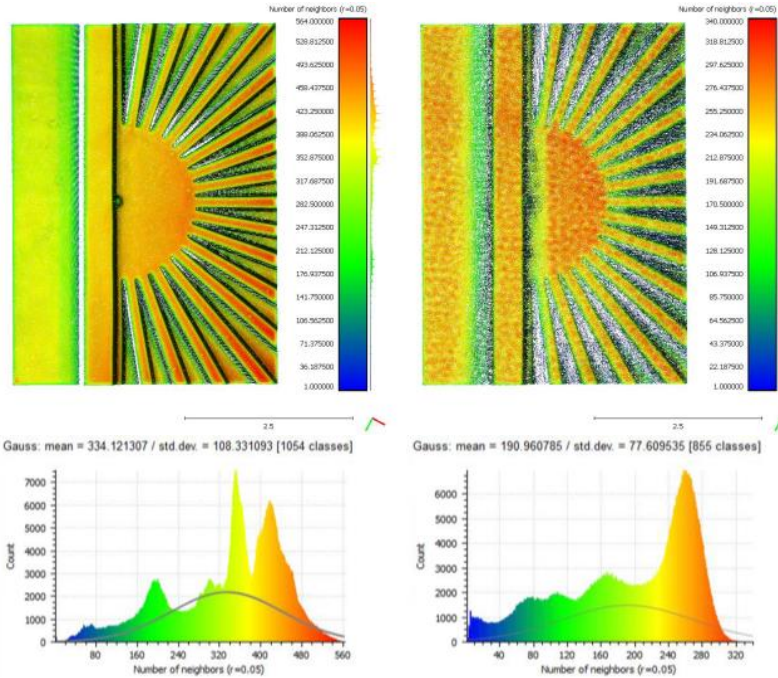

Figure 8. Upper part of the apse, density comparison between TLS (left) and MMS (right). The analysis has been performed using CC (number of neighbour analysis, pts/V sphere $\mathrm{r}=0.05 \mathrm{~m}$ ).
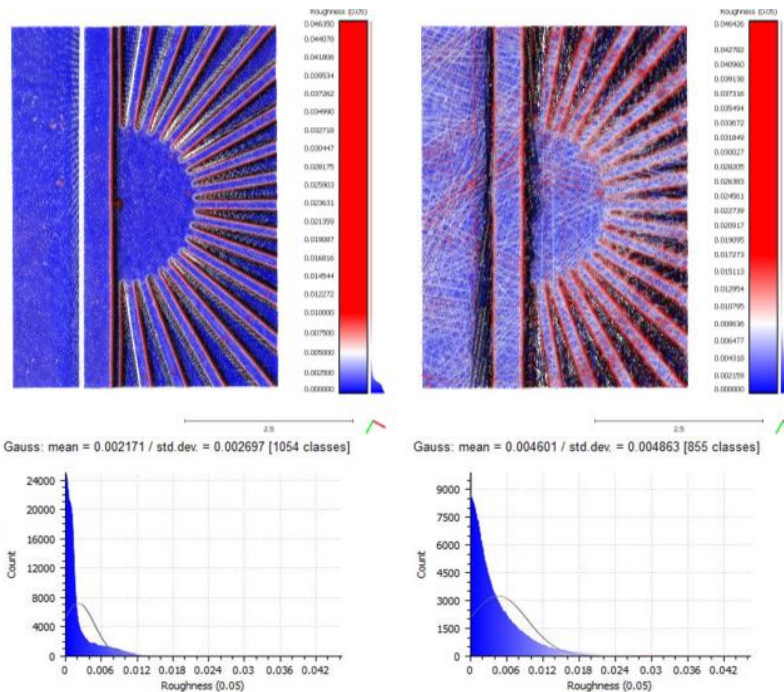

Figure 9. Upper part of the apse, roughness comparison between TLS (left) and MMS (right). The analysis has been performed using CC $(\mathrm{di} / \mathrm{V}$ sphere $\mathrm{r}=0.05 \mathrm{~m})$. 


\subsubsection{Planar surface analysis}

The second example regards a planar surface, therefore a portion of a wall located in the apse of the pavilion has been analysed. Also in this case the TLS density (mean value: ca 614 points/V sphere $r=$ $0.05 \mathrm{~m}$ ) is higher than Swift density (mean value: ca 193 points/V sphere $r=0.05 \mathrm{~m}$ ).

As expected, the TLS point cloud appears to be denser in the area closer to the laser scanner (in this case, in the area on the left of the wall, where the device was positioned); instead, the Swift System data is more homogenous, since the operator during the acquisition has walked at a constant distance from the wall and at a constant speed. However, as previously said, it is possible to observe some areas characterised by a regular pattern with a discontinuous level of density. This characteristic is particularly visible in Figure 10 and in the roughness analysis displayed in Figure 11.
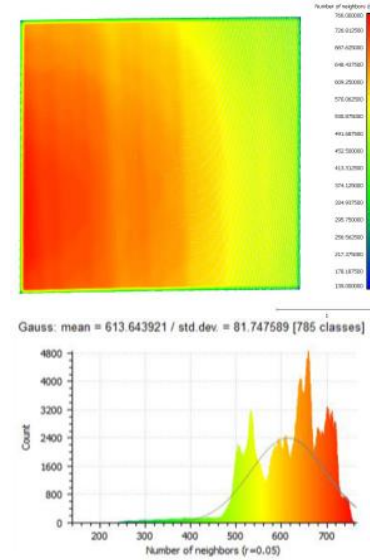
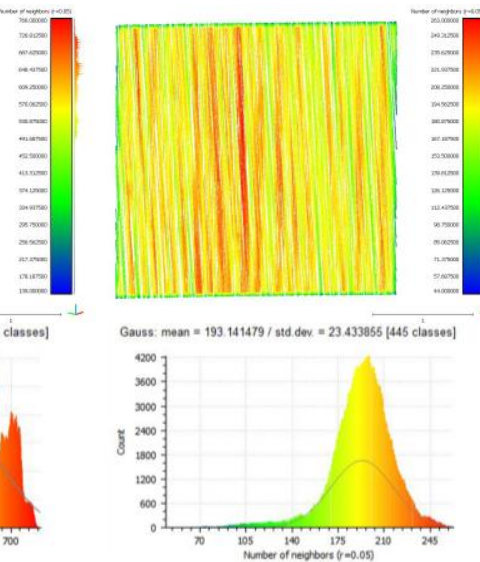

Figure 10. Analysis of a wall portion as planar surface: density comparison between TLS (left) and MMS (right). The analysis has been performed using CC (number of neighbour analysis, $\mathrm{pts} / \mathrm{V}$ sphere $\mathrm{r}=0.05 \mathrm{~m})$.
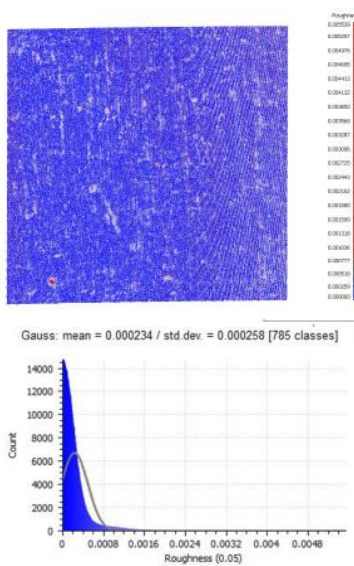
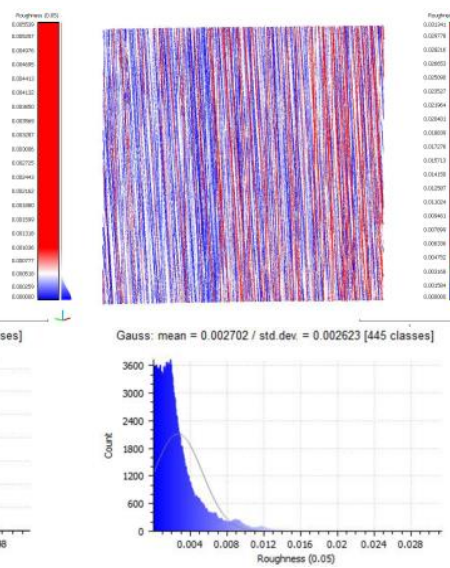

Figure 11. Analysis of a wall planar surface: roughness index comparison between TLS (left) and MMS (right). The analysis

has been performed using CC $(\mathrm{di} / \mathrm{V}$ sphere $\mathrm{r}=0.05 \mathrm{~m})$.

Also as concerns the portion of the considered wall, the roughness value is higher, as in the previous case of the vault, in the MMS scan (mean value: ca $0.003 \mathrm{~m}$ ) compared to the ca $0.0002 \mathrm{~m}$ observed in the Faro Focus ${ }^{3 \mathrm{D}}$ X330 point cloud. The highest levels of roughness correspond to the acquisition profiles of the laser beam of the Swift system which generate a corrugation on the surface of the acquired object and, therefore, noise. This is probably due to the vibrations of the trolley during the acquisition of the $3 \mathrm{D}$ point cloud.

\subsubsection{The detail of the Agnelli statue}

In the third and last example - the statue of Giovanni Agnelli the density is significantly higher in the Swift point cloud (mean value: ca 835 points $/ \mathrm{V}$ sphere $\mathrm{r}=0.05 \mathrm{~m}$ ) (Figure 12). Also considering the relatively high standard deviation (due to the elevate density difference in the central part of the niche where the point cloud is sparser, caused by the partial occlusion of the statue), in this case the MMS data is denser than LiDAR data (mean value: ca 258 points/ $\mathrm{V}$ sphere $\mathrm{r}=0.05 \mathrm{~m}$ ). That can be explained by the higher distance between the TLS stations and the statue during the acquisition (between 8 and $18 \mathrm{~m} \mathrm{ca}$ ), in comparison to the Swift System which instead moved very close to the statue (trajectory A) (Figure 4) for a longer time, collecting a denser data. As already evident in the previous example, also in the case of the statue point cloud a relatively high level of noise $(\approx 3 \mathrm{~mm})$ is observable in correspondence with the profile of the laser (Figure 13);
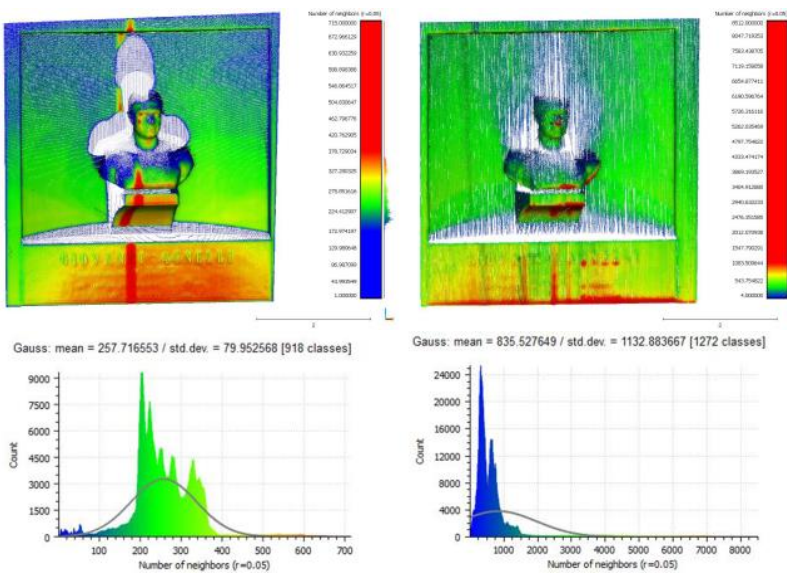

Figure 12. Giovanni Agnelli statue: density comparison between TLS (left) and MMS (right). The analysis has been performed using $\mathrm{CC}$ (number of neighbour analysis, pts/V sphere $r=0.05 \mathrm{~m})$.
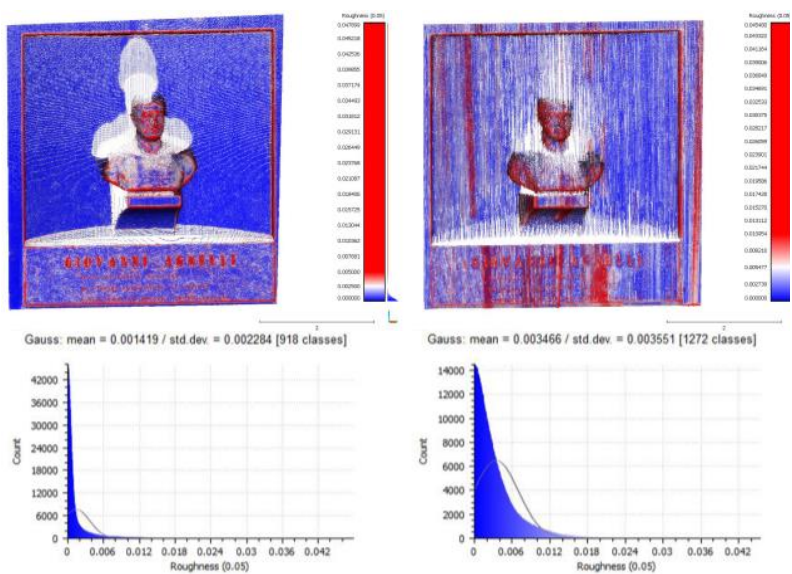

Figure 13. Agnelli bust statue: roughness comparison between TLS (left) and MMS (right). The analysis has been performed using CC $(\mathrm{di} / \mathrm{V}$ sphere $\mathrm{r}=0.05 \mathrm{~m})$.

The level of detail is lower in comparison to the model achieved by the LiDAR system, where the finer details are visually more appreciable, and the geometry of the statue is most completely reconstructed; However, the statue has been acquired by the Swift system with an adequate resolution and, in addition, thanks to free motion allowed by the movable solution represented by the trolley, the lack of data behind the statue due to occlusions is significantly lower. 


\subsubsection{Profiles misalignments and topological errors}

Both the test-cases offer the chance of evaluating an error occurrence phenomenon for the Swift trolley affecting the uncertainty of the metric product, that it is now possible to say frequent due to the mechanical system functioning itself. The alignment of profiles, related to the SLAM algorithm, is also sensitive to the ScanPlan configuration and much to the inertial sensor equipping the device. Non-planar/levelled surfaces or slightly sloping floors can affect the global registration and FARO Technologies first suggests acquiring data only on horizontal surfaces. As occurred in the Cinema, the pavements trends, in fact, and the slight slope of parterre pavements can cause problems in the functioning of the SLAM and the drift errors affecting the trajectory are sensitive. It is also possible to notice in the statue bust detail some outliers and topological errors due to the vibrations caused by the trolley and rubble during operator movement (Figure 15).

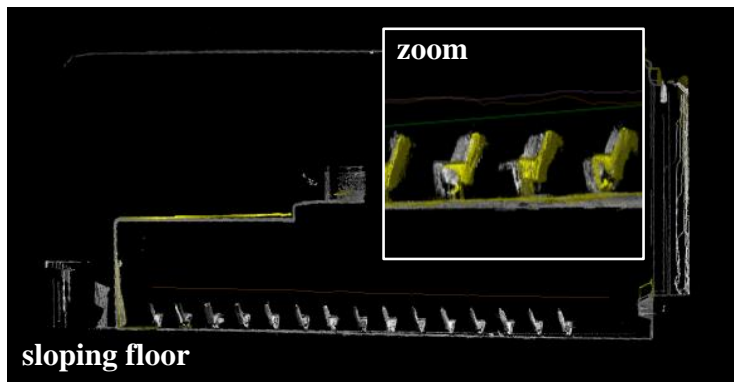

Figure 14. The drift error in the cinema parterre, with the sloping floor

(a)

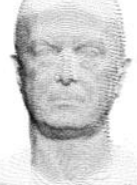

(b)

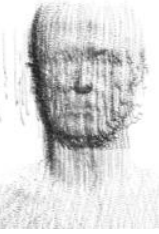

Figure 15. Some topological errors on the Agnelli bust statue due to the vibrations of the trolley in (a) TLS and (b) MMS.

\section{CONCLUSIONS AND FUTURE PERSPECTIVES}

The market for advanced solutions devoted to massive digitisation has certainly developed devices that greatly optimize the manoeuvrability and speediness of the systems, focusing on the suitability to specific application fields. The Swift system by FARO certainly intends to target the application field of digitisation projects of extended environments characterised by standard building elements of large complexes (airports, hospitals, office blocks). Here the historical structures legacies of $20^{\text {th }}$ century have been used as test dataset. With respect to the relevant results from preliminary data it is necessary to underline the positive reception of the outcomes and also to notice some bottlenecks of the system operation. We intend also to estimate time-costs balancing and prove that even heritage environments, for example the architectures characterised by enormous vaulted systems such as the ferrocement spatial structures of the $20^{\text {th }}$ century, can be efficiently digitised with these systems. On the other hand, the manageability of the huge point clouds datasets deriving from the Swift system (roughly $10 \mathrm{~Gb}$ per scans that should be necessarily filtered and decimated), as introduced, is actually due to the movement of a long range static scanner (S350 series). During these first tests, it has been surely under discussion the key-issue of the scarce possibility for the operator to interact with results as a drawback, especially in the postprocessing phase with the proprietary software interface, for example with the possibility of classifying the clouds with respect to the acquisition time. The very recent release of a new FARO Focus $S$ firmware (v. 6.8) and software version (SCENE 2021), that introduce time and movements filters during post-processing phase to develop the Swift System functionalities, is permitting us to have a second test and evaluate the new improved capabilities.

\section{ACKNOWLEDGEMENTS}

The tests have been possible thanks to the gentle cooperation of the CAM2 (FARO Technologies, Inc.) AEC Team Italy . The authors would like to greatly thank A. Sardo and L. Mattioli. The Turin Exhibition Centre conservation project, to which the complete 3D survey is related, is funded by Keeping it Modern - Planning Grant 2019 of the Getty Foundation

\section{REFERENCES}

Barba, S., Ferreyra, C., Cotella, V. A., Filippo, A. di, \& Amalfitano, S. (2021). A SLAM Integrated Approach for Digital Heritage Documentation. In: Rauterberg, M. (Ed), Culture and Computing. Interactive Cultural Heritage and Arts, 27-39.

Betti, M., Bonora, V., Galano, L., Pellis, E., Tucci, G., \& Vignoli, A. (2021). An Integrated Geometric and Material Survey for the Conservation of Heritage Masonry Structures. Heritage, 4(2), 585-611.

Calantropio, A., Matrone, F., \& Lingua, A. M. (2019). L'integrazione di tecniche fotogrammetriche e MMS nel rilievo metrico dei Beni Culturali. Atti ASITA 2019, 157-164.

Di Filippo, A., Sánchez-Aparicio, L., Barba, S., Martín-Jiménez, J., Mora, R., \& González Aguilera, D. (2018). Use of a Wearable Mobile Laser System in Seamless Indoor 3D Mapping of a Complex Historical Site. Remote Sensing, 10(12), 1897.

Di Pietra, V., Grasso, N., Piras, M., \& Dabove, P. (2020). Characterization of a Mobile Mapping System for seamless navigation. The International Archives of the Photogrammetry, Remote Sensing and Spatial Information Sciences, XLIII-B1-2, 227-234.

Fuentes-Pacheco, J., Ruiz-Ascencio, J., \& Rendón-Mancha, J. M. (2012). Visual simultaneous localization and mapping: a survey. Artificial Intelligence Review, 43(1), 55-81.

Masiero, A., Fissore, F., Guarnieri, A., Piragnolo, M., \& Vettore, A. (2017). Comparison of low cost photogrammetric survey with TLS and Leica Pegasus Backpack 3D modelss. The International Archives of the Photogrammetry, Remote Sensing and Spatial Information Sciences, XLII2/W8, 147-153.

Nocerino, E., Menna, F., Remondino, F., Toschi, I., \& RodríguezGonzálvez, P. (2017). Investigation of indoor and outdoor performance of two portable mobile mapping systems. In: F. Remondino \& M. R. Shortis (Eds.), Videometrics, Range Imaging, and Applications XIV, SPIE 2017, 103320I.

Puente, I., González-Jorge, H., Martínez-Sánchez, J., \& Arias, P. (2013). Review of mobile mapping and surveying technologies. Measurement, 46(7), 2127-2145.

Remondino, F., Gruen, A., von Schwerin, J., Eisenbeiß, H., Rizzi, A., Girardi, S., Sauerbier, M., \& Richards-Rissetto, H. (2009). Multi-sensor 3D documentation of the Maya site of Copan. Proceedings of $22^{\text {nd }}$ CIPA Symposium, Kyoto, Japan.

Sammartano, G., \& Spanò, A. (2018). Point clouds by SLAM-based mobile mapping systems: accuracy and geometric content validation in multisensor survey and stand-alone acquisition. Applied Geomatics, 10(4), 317-339.

Riisgaard, S. \& Blas, M. R. (2005). SLAM for Dummies. A Tutorial Approach to Simultaneous Localization and Mapping. MIT OCW.

Toschi, I., Rodríguez-Gonzálvez, P., Remondino, F., Minto, S., Orlandini, S., \& Fuller, A. (2015). Accuracy evaluation of a Mobile Mapping System with advanced statistical methods. The International Archives of the Photogrammetry, Remote Sensing and Spatial Information Sciences, XL5/W4(5W4), 245-253.

Tucci, G., Visintini, D., Bonora, V., \& Parisi, E. (2018). Examination of Indoor Mobile Mapping Systems in a Diversified Internal/External Test Field. Applied Sciences, 8(3), 401.

Zhang, J., \& Singh, S. (2018). Laser-visual-inertial odometry and mapping with high robustness and low drift. Journal of Field Robotics, 35(8), 12421264. 